\title{
CFD heat transfer simulation of the human upper respiratory tract for oronasal breathing condition
}

\author{
Kambiz Farahmand* , Raghavan Srinivasan, and Mohsen Hamidi
}

Department of Industrial and Manufacturing Engineering, North Dakota State University, Dept. 2485, PO Box 6050, Fargo, ND 58108-6050, USA

\begin{tabular}{l}
\hline A R T I C L E I N F O \\
\hline Article history: \\
Received 1 August 2011 \\
Available online \\
10 August 2011 \\
\hline Keywords: \\
Oronasal breathing \\
Heat transfer simulation \\
Human upper respiratory tract
\end{tabular}

\section{Introduction}

Various studies have investigated heat and mass transfer in the HRT. The inspired air is heated to normal body temperature, and the expired air is cooled to regain the heat back in the body. These measurements of heat and water transport were carried out by the use of thermocouples (Farahmand and Kaufman 2006). Mathematical models depicting the heat and water transport phase (McCutchan et al. 1951, Tsai et al. 1990 and Tsu et al. 1988) were used for numerical simulation and to determine the heat transfer characteristics of the region. Recent trends include generating the three- dimensional (3D) model of the respiratory tract into CFD software. The model generation is based on the magnetic resonance imaging (MRI) or computed axial tomography (CAT) scan of the respiratory tract obtained from a healthy volunteer. Most models use the Navier-Stokes equation for the CFD simulation. The

* Corresponding author Tel +1-701-231-5694; fax: +1-701-231-7195

E-mail: Kambiz.Farahmand@ndsu.edu (K. Farahmand)

¿ 2012 Growing Science Ltd. All rights reserved. doi: 10.5267/j.ijiec.2011.08.017 
simulation performed in these cases analyzed the heat transfer at room temperature. The study of the heat and mass transfer, aerosol deposition, and flow characteristics in the upper HRT using computational fluid mechanics simulation requires access to a two-dimensional (2D) or 3D model of the HRT. An exact model is complex since it involves the use of imaging devices on the human body; therefore, a simplied 3D geometry representing the upper HRT is developed, consisting of the nasal cavity, oral cavity, nasopharynx, pharynx, oropharynx, trachea, and the first two generations of the bronchi.

The respiratory tract is modeled circular in cross-section and in varying diameter for various portions as identified in this study. The dimensions are referenced from the literature herein. Based on the dimensions, a simplified 3D model, representing the upper HRT, is generated. This model will be useful in studying the flow characteristics and could assist in treament of injuries of the HRT as well as help determine a drug delivery mechanism and dosages.

A study of the heat transfer mechanism of the HRT helps assess any heat, smoke, and fire-related injury affecting the HRT. The design of respirator systems used by people working in extreme environments, like firefighters exposed to forest fires, chemical and biological hazards, or hazardous materials, can be better improved by comprehensive study of the thermal profile. This can help create better occupational health and safety in the case of firefighters and emergency responders. These emergency responders are exposed to extreme temperatures, and although they do have protective equipment such as respirators for oxygen supply, they still inhale air that is heated because of the extreme temperature in the surrounding atmosphere. Lv et al. (2006) evaluated burn injury due to inhalation of hot gas. In emergency situations, there is bound to be chaos and confusion in addition to the physical load; the increased heart rate and respiration rate dictate a high possibility of oronasal breathing (simultaneous breathing via oral and nasal cavity) taking place expectedly when under physical stress. A 3D heat-transfer study is performed here to identify the temperature obtained at the surface of the tissue during the inspiration phase. Literature is available that studies the temperature profile in the respiratory tract, including the nasal and the oral respiratory tract separately.

\section{Methodology}

To study characteristics such as particle deposition, burn injury, and heat and mass transfer in the upper HRT, various models were developed. These models were based on images obtained from computed tomography (CT) scans, MRI, and/or acoustic rhinomanometry (AR). This procedure is complex, and access to these images is limited. The cost involved with the use of these data too is high; therefore, simplified airway geometry of the HRT, consisting of the nasal airway, oral airway, pharynx, trachea, and the first two generations of the bronchi, is developed based on the data available from the literature cited herein.

Simplified models that could be standardized for the purpose of CFD simulation were developed and used in this study. The geometry of the respiratory tract is complex and hugely different for each individual. The model developed here is based on the data available in literature cited herein (Cheng et al. 1996, Cheng et al. 1997, Erthbruggen et al. 2004, Gemci et al. 2002, Grgic et al. 2004, Inthavong et al. 2006, Johnstone et al. 2004, Liu et al. 2009, Robinson et al. 2009, Wang et al. 2009, Weibel 1963, Wen et al. 2008, Xi et al. 2008, Yu et al. 1996, Zhang, Z. and Kleinstreuer 2003), as measurement of real-time data is a complex process and costly. Based on the measurement in the literature, the simplified 3D geometry of the upper HRT is developed. The proposed method here is to build a simplified three-dimensional model representing the upper HRT that serves as a simplified model to simulate and study various flow characteristics. 
Fig. 1 shows the structure of the HRT, consisting of the nasal cavity, oral cavity, nasopharynx, pharynx, larynx, trachea, and first two generations of the bronchi. The geometry here has varying diameter for the nasal, oral, pharynx, larynx, tracheal, and bronchi regions.

Human tissue is highly flexible; it contracts and expands based on the amount of pressure applied on the human body. The pressure exerted by body posture also leads to the expansion or contraction of the internal organs including the HRT. For example, when a person is lying on his or her back, there is expansion in the human body, and while bending forward, there is a contraction of the muscles. These complicated factors contribute to the complexity of defining the geometry of the respiratory tract. The simple model created here represents an ideal HRT up to the first two generations of the bronchi.

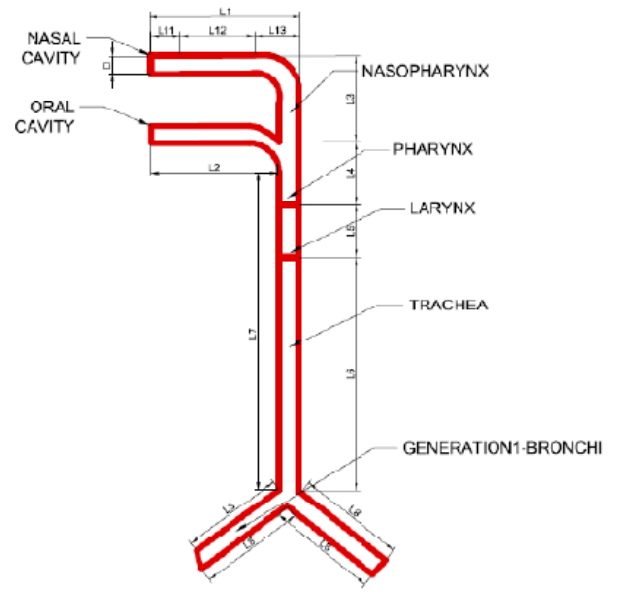

Fig. 1. The Human respiratory tract

\section{CFD Simulation}

CFD software enables us to study heat transfer characteristics (temperature profile) and performs calculations using numerical formulas/equations based on the laws of physics. The CFD simulation is based on the Navier-Stokes equation for three-dimensional incompressible flows. The CFD software used here for simulation is Ansys CFX 12. The procedure to use the CFD software generally consists of four major steps. First, the geometry is created using three-dimensional modeling software. After development, the model is imported into the CFD software; this constitutes the second step where parameters are defined. The third step is the simulation run, and the fourth step consists of the postprocessing of the results.

Air intake in the respiratory tract will take place in an excited condition where oronasal breathing takes place in emergency situations. The volume of air intake in this case will be subject to oronasal breathing during heavy exercise. Wheatley et al. (1991) have determined airflow characteristics during heavy exercise and have stated that over $80 \%$ of normal subjects breathe oronasally. The minute ventilation $\left(V_{E}\right)$ before the exercise was $10.7+/-1.01 \mathrm{l} / \mathrm{min}$. The switch from nasal to oronasal breathing took place at a minute ventilation of $22.3+/-3.5 \mathrm{l} / \mathrm{min}$, and the final value obtained was $75.7+/-5.01 \mathrm{l} / \mathrm{min}$. Malarbet et al. 1994 observed that the switch from nasal to oronasal was made at the ventilation rate of $35 \mathrm{l} / \mathrm{min}$, and the maximum observed value of ventilation rate was $90 \mathrm{l} / \mathrm{min}$. The minute ventilation or total ventilation $\left(V_{E}\right)$ is the product of tidal volume $\left(V_{T}\right)$ and frequency of breathing $(f)$. This is mathematically expressed as $V_{E}=V_{T}$. $f$ where $V_{T}$ is the volume of air exhaled during one respiratory cycle.

The fluid breathed in during the inspiration phase consists of hot air having a temperature of $100^{\circ}$ Celsius, the density of the air being $0.946 \mathrm{~kg} / \mathrm{m}^{3}$. The wall is maintained at a temperature of $37^{\circ}$ Celsius with the heat transfer option selected. The fluid flow is a turbulent flow, having a high 
Reynolds number. Varene et al. (1986), have conducted a study concluding that the temperature during inspiration $\left(\mathrm{T}_{\mathrm{I}}\right)$ and during expiration $\left(\mathrm{T}_{\mathrm{E}}\right)$ differs for the oral and nasal cavities (the oral cavity having the higher temperature rate than the nasal cavity). But it was concluded by that no large differences exist from an energetic point of view between the nasal and oral cavities. The differences in heat exchange were found to be less, and the total power loss was only $7 \%$ lower during nasal breathing than during mouth breathing. Niinima et al. (1981) also studied the effect of exercise on breathing. It was concluded that as the intensity of exercise increased, the flow rate increased. The Reynolds number calculated here is 4130 , based on the diameter of the trachea. The convergence criterion was set to $1 \mathrm{E}-6$ and the maximum number of iterations was set to 200 . Two different mesh configurations were considered for the study.

\section{Results}

The results from the simulation were obtained for temperature distribution along the flow for the nasal cavity, oral cavity, and the trachea. A cross-sectional view showing the temperature variation inside the respiratory tract up to the tracheal region is shown in Fig. 2.

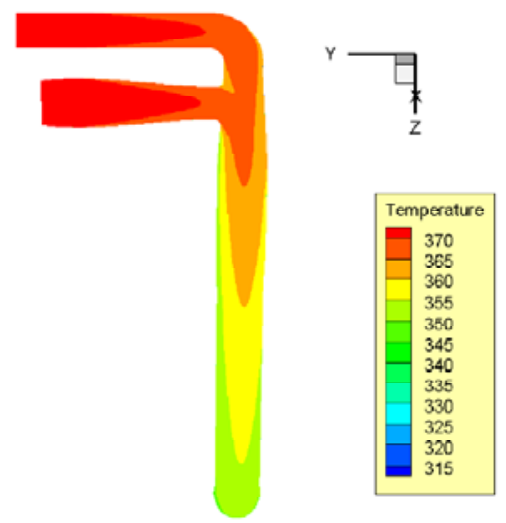

Fig. 2. Temperature variation (A cross sectional view, temperature in $\mathrm{K}$ )

The temperature variations can be observed here. At the inlet, the temperature is the air temperature (surrounding temperature), and the air temperature decreases as it flows through the tract. This is due to the heat exchange between the inlet air and the respiratory tract. Temperature profiles for the portion of the nasal cavity, oral cavity, and the tracheal region along the center, as the path along which temperature variation is shown, is identified as A) for nasal cavity, B) for oral cavity, and C) for tracheal region in Fig. 3.

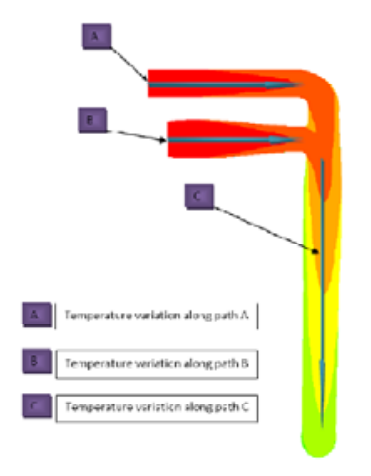

Fig. 3. Temperature profile along the nasal, oral, and trachea

Fig. 4 depicts the variation in temperature along the center path of the nasal cavity up to a distance of $100 \mathrm{~mm}$ in a horizontal direction (part A in Fig. 3). The variations in temperature can be seen for the 
two different mesh configurations. A decrease in the temperature of air is observed as air flows from the inlet of nasal and oral cavity through the tracheal region.



Fig. 4. Nasal temperature profile for two mesh configurations

Fig. 5 shows the variation in temperature along the center path of the oral cavity in a horizontal direction (part B in Fig. 3). The variations in temperature can be seen for the two different mesh configurations. A decrease in the temperature of air is observed as the distance increases.

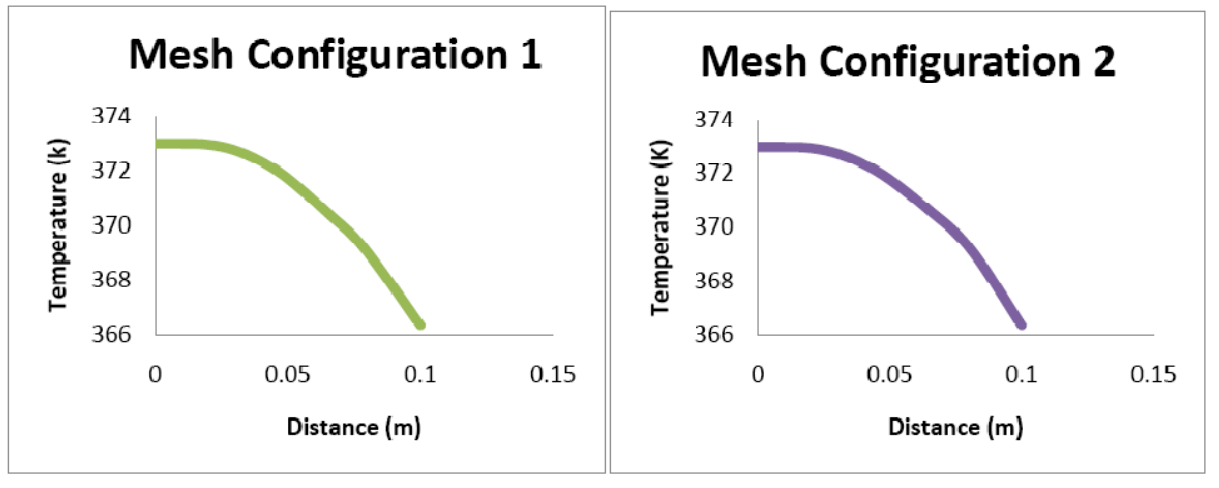

Fig. 5. Oral Temperature Profile

Fig. 6 depicts the variation in temperature along the center path of the trachea up to a distance of 200 $\mathrm{mm}$ in a vertical direction (part $\mathrm{C}$ in Fig. 3). The variations in temperature can be seen for the two different mesh configurations. A decrease in the temperature is observed as the flow reaches close to the bronchial region.



Fig. 6. Tracheal Temperature Profile

The inlet air temperature decreases gradually as it passes through the bronchi to the lungs. This phenomenon is due to the fact that the body absorbs much of the heat from the inlet air if the inlet air 
temperature is higher than the body temperature. As it is assumed that the body temperature is constant throughout and less than the inlet air temperature, it is expected that there will be a decrease in the air temperature as it reaches the bronchi.

The temperature is due to the flow along the wall surface being in range of $315^{\circ} \mathrm{K}$ for the two meshes. At the center along the centerline the temperature is high, which is the inlet atmospheric air at $373^{\circ} \mathrm{K}$ as in Fig. 7. The temperature profile along the center of the respiratory tract is observed for the specified path in Figures 2, 3, 4, 5, and 6.


Fig. 7. Surface temperatures obtained

Fig. 8 compares the temperature profile along the three different types identified as oronasal breathing, nasal breathing, and oral breathing conditions.

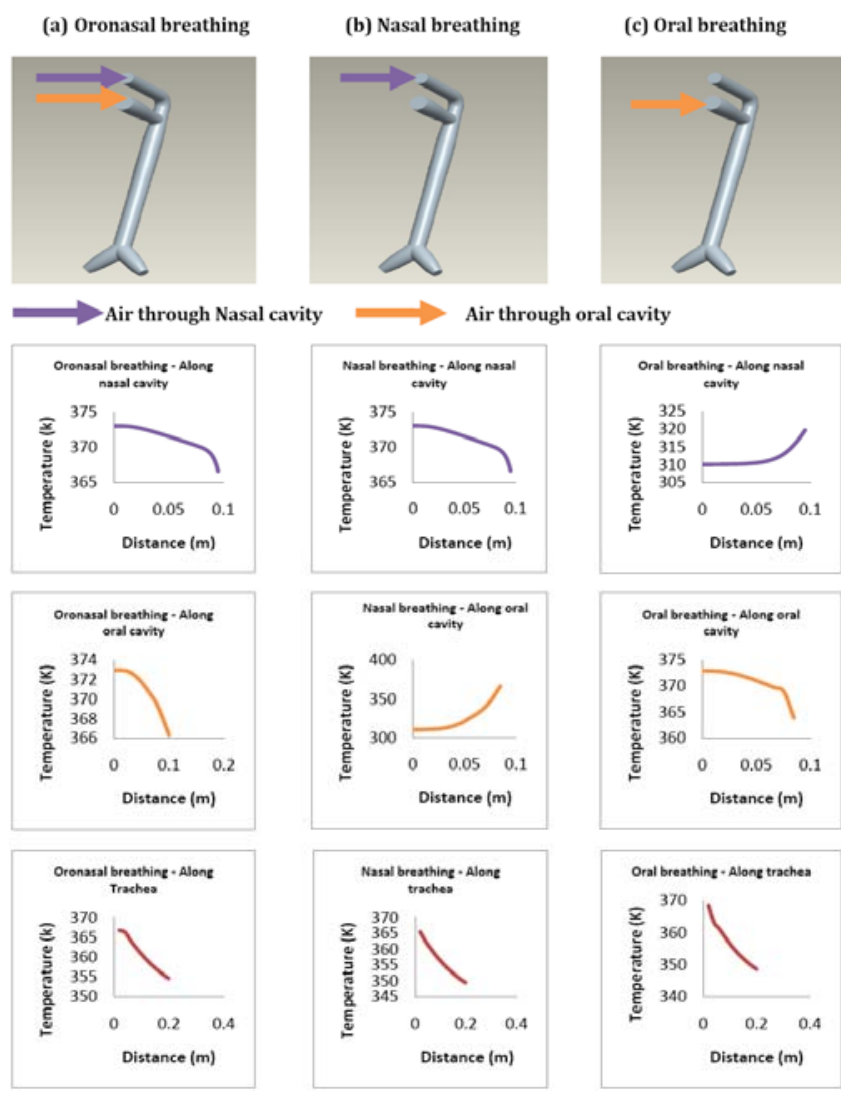

Fig. 8. Comparison of Nasal Breathing, Oral Breathing, and Oronasal Breathing 
The profile for the nasal cavity during oronasal breathing and nasal breathing follows a similar trend. The inlet temperature here is $373^{\circ} \mathrm{K}$ at a distance of $100 \mathrm{~mm}$ it drops to $366^{\circ} \mathrm{K}$. For oral breathing, the temperature profile has an upward trend. The profile for the oral cavity during oronasal and oral breathing follows a similar pattern where the inlet temperature is $373^{\circ} \mathrm{K}$, and the temperature obtained at a distance of $100 \mathrm{~mm}$ is about $364^{\circ} \mathrm{K}$. The tracheal temperature profile for the three different breathing conditions has a similar trend, the temperature at the inlet point of the path being about $365-368^{\circ} \mathrm{K}$ and decreasing to $350-355^{\circ} \mathrm{K}$ for a distance of $200 \mathrm{~mm}$ as defined in the model.

\section{Conclusion}

The temperature plots shown in Fig. 4, Fig. 5, and Fig. 6 give temperature variations along the nasal cavity, oral cavity, and the tracheal region. For the nasal and oral cavities, the temperature variation shown starts from the inlet portion up to a distance of $100 \mathrm{~mm}$ inside the cavity. The temperature variation for the tracheal cavity is from the start of the oropharynx region up to a distance of $200 \mathrm{~mm}$ close to the bronchi. This temperature change is represented by the points along the center of the cavity. The temperature of the walls for the two mesh configurations in Figure 7 shows a temperature of $315^{\circ} \mathrm{K}$. Thus it can be inferred that the inhaled air causes the wall temperature of the respiratory tract to attain a temperature of $315^{\circ} \mathrm{K}$ when oronasal breathing takes place in the conditions specified. For the mesh validation, two different mesh configurations were simulated. The two mesh sizes give similar results; therefore, we can conclude that the results are independent of the grid size. This wall temperature will be the temperature obtained at the surface of the tissue, leading to a possible injury to the respiratory tract.

A heat-transfer study along the HRT gives the temperature distribution and variation along the surface of the respiratory tract for the given length during oronasal breathing conditions. Obtaining the temperatures along the tissue walls helps in assessing the internal burn injury. This study will assist in developing safe and effective preventive measures and treatments for the injuries caused in the respiratory tract. Design of respirator devices and safety features for occupations that involve exposure to extreme and unfavorable conditions hazardous to human health can be well implemented by knowing the level of injury caused in the HRT. A comparison of the differences in the temperature profile in the HRT considering variations in breathing patterns and the implementation of respiratory devices to cool the inhaling air temperature to normal range will be quite effective in preventing any injury. Future studies could include the use of hazardous particle deposition along the respiratory tract during oronasal breathing while simulating various adverse conditions.

\section{References}

Cheng, K. H., Cheng, Y. S., Yeh, H. C., Guilmette, R. A., Simpson, S. Q., Yang, Y., \& Swift, D. L. (1996). In VIVO measurement of nasal airway dimensions and ultrafine aerosol deposition in the human nasal and oral airways. Journal of Aerosol Science, 27(5), 785-801.

Cheng, K. H., Cheng, Y. S., Yeh, H. C., \& Swift, D. L. (1997). Measurements of airway dimensions and calculation of mass transfer characteristics of human oral passage. Transactions of the ASME, 119, 476-482.

Erthbruggen, C., Hirsch, C., \& Paiva, M. (2004). Anatomically based three dimensional model of airways to simulate flow and particle transport using computational fluid dynamics. Journal of Applied Physiology, 98, 970-980.

Farahmand, K., \& Kaufman, J. W. (2006). In vivo measurements of human oral cavity heat and water vapor transport. Respiratory Physiology \& Neurobiology, 150, 261-277.

Gemci, T., Corcoran, T.E., \& Chigier, N. (2002). A numerical and experimental study of spray dynamics in a simple throat model. Aerosol Science and Technology, 36, 18-38.

Grgic, B., Finlay, W. H., \& Heenan, A. F. (2004). Regional aerosol deposition and flow measurements in an idealized mouth and throat. Journal of Aerosol Science, 35, 21-32. 
Grgic, B., Finlay, W. H., Burnell, P. K. P., \& Heenan. A. F. (2004). In vitro intersubject and intrasubject deposition measurements in realistic mouth-throat geometries. Journal of Aerosol Science, 35, 1025-1040.

Inthavong, K., Tian, Z. F., Li, H. F., Tu, J. Y., Yang, W., Xue. C. L., \& Li, C. G. (2006). A numerical study of spray particle deposition in a human nasal cavity. Aerosol Science and Technology, 40, 1034-1045.

Johnstone, A., Uddin, M., Pollard, A., Heenan, A., \& Finlay, W. H. (2004). The flow inside an idealized form of the human extra-thoracic airway. Experiments in Fluids, 37, 673-689.

Liu,Y., Johnson, M. R., Matida, E. A., Kherani, S., \& Marsan, J. (2009). Creation of standardized geometry of the human nasal cavity. Journal of Applied Physiology, 106, 784-795.

Lv, Y. G., Liu, J., \& Zhang, J. (2006). Theoretical evaluation of burns to the human respiratory tract due to inhalation of hot gas in the early stage of fires. Journal of the International Society for Burn Injuries, 32, 2006, 436-446.

Malarbet, J. L., Bertholon, J. F., Becquemin, M. H., Taieb, G., Bouchikhi, A., \& Roy, M. (1994). Oral and nasal flowrate partitioning in healthy subjects performing graded exercise. Radiation Protection Dosimetry, 53, 179-182.

McCutchan, J. W., \& Taylor, C. L. (1951). Respiratory Heat Exchange with Varying Temperature and Humidity of Inspired Air. Journal of Applied Physiology, 4, 121-135.

Niinimaa, V., Cole, P., Mintz, S., \& Shephard, R. J. (1981). Oronasal distribution of respiratory airflow. Respiration Physiology, 43, 69-75.

Robinson, J. R., Russo, J., \& Doolittle, R. (2009). 3D Airway reconstruction using visible human data set and human casts with comparison to morphometric data. The Anatomical Record, 292, 10281044.

Tsai, C. L., Saidel, G. M., McFadden, E. R., \& Fouke, J.M. (1990). Radial heat and water transport across the airway wall. The American Physiological Society, 69 (1), 222-231.

Tsu, M., Babb, A. L., Ralph, D. D., \& Hlastala, M. P. (1988). Dynamics of heat, water and soluble gas exchange in the human airways: 1. A model study. Annals of Biomedical Engineering, 16, 547-571.

Varene, P., Ferrus, L., Manier, G., \& Gire, J. (1986). Heat and water respiratory exchanges: comparison between mouth and nose breathings in humans. Clinical Physiology, 6, 405-414.

Wang, Y., Liu, Y., Sun, X., Yu, S., \& Gao, F. (2009). Numerical analysis of respiratory flow patterns within human upper airway. Acta Mech Sin.

Weibel, E. R. (1963). Morphometry of Human Lung. Academic, New York.

Wen, J., Inthavong, K., Tu, J., \& Wang, S. (2008). Numerical simulations for detailed airflow dynamics in a human nasal cavity. Respiratory Physiology and Neurobiology, 161, 125-135.

Wheatley, J. R., Amis, T. C., \& Engel, L. A. (1991). Oronasal partitioning of ventilation during exercise in humans. The American Physiological Society, 71 (2), 546-551.

Xi, J., \& Longest, P. W. (2008). Effects of oral airway geometry characteristics on the diffusional deposition of inhaled nanoparticles. Journal of Bio-mechanical Engineering, 130, 1-16.

Yu, G., Zhang, Z., \& Lessman, R. (1996). Computer simulation of the flow field and particle deposition by diffusion in a 3-D human airway bifurcation. Aerosol Science and Technology, 25, 338-352.

Zhang, Z., \& Kleinstreuer, C. (2003). Species heat and mass transfer in a human upper airway model. International Journal of Heat and Mass Transfer, 46, 4755-4768. 